obtained lower results $(A \cup C=0.60$, cutoff $=2.38$ ). Figure 1 shows cumulative probability plots of mSASSS and ASDAS versus OWD, showing cutoff values determined by ROC analysis.
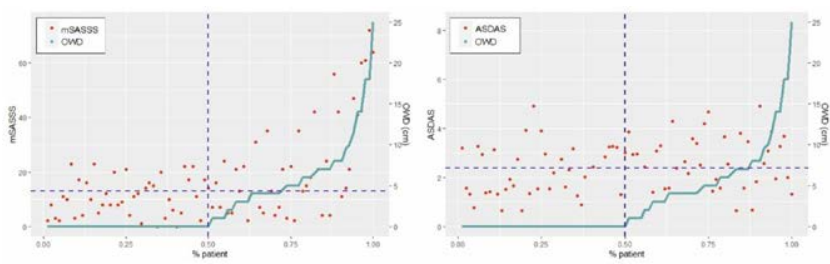

Conclusion: Structural damage, in cervical and lumbar spine, are the main contributor to HK appearance in axSpA patients. Inflammation it is not so determinant and acts only in the short term. HK produces a significant reduction of cervical mobility and physical function. New treatments that decrease radiographical progression, and overweight reduction are the most important factor to avoid HK.

REFERENCES:

[1] Measuring Spinal Mobility Using an Inertial Measurement Unit System: A

Validation Study in Axial Spondyloarthritis. Diagnostics 2020, 10(6), 426.

Disclosure of Interests: None declared

DOI: 10.1136/annrheumdis-2021-eular.346

\section{POS0949 HIGH PREVALENCE OF ABDOMINAL OBESITY IN FEMALES WITH AXIAL SPONDYLOARTHROPATHY}

S. Maguire ${ }^{1,2}$, P. Gallagher ${ }^{3}$, F. Wilson ${ }^{4}$, F. B. O'shea ${ }^{1,2}$. 'St James' Hospital, Rheumatology, Dublin, Ireland; ' $T$ Trinity College Dublin, School of Medicine, Dublin, Ireland; ${ }^{3}$ St Vincent's Hospital, Rheumatology, Dublin, Ireland; ${ }^{4}$ Trinity College Dublin, Discipline of Physiotherapy, Dublin, Ireland

Background: Worldwide prevalence of obesity has been steadily increasing, despite significant public health campaigns to raise health awareness. In axial spondyloarthropathy (axSpA) obesity has been shown to be associated with higher levels of disease activity and decreased response to treatment. The waist to hip ratio (WtHpR) is a tool to screen for abdominal obesity. Abdominal deposition of adipose tissue is associated with increased risk of cardiovascular disease, type II diabetes and premature death. Abdominal obesity is more commonly found in males, while females are more prone to gluteal-femoral fat deposition. The Ankylosing Spondylitis Registry of Ireland (ASRI) is a source of epidemiological data of axSpA patients in Ireland.

Objectives: To capture the prevalence of abdominal obesity in a large cohort of axSpA patients and assess for variation in prevalence between sexes.

Methods: Participants were analysed on the basis of sex and presence of obesity by $\mathrm{BMI}$ and WtHpR. Obesity was defined by body mass index (BMI) with a result of $>30$ categorised as obese as per CDC definitions. Abdominal obesity was assessed by $\mathrm{WtHpR}$ and defined as per WHO guidelines. Categorical variables were recorded as frequencies, with a chi ${ }^{2}$ test for independence used to test for statistical significance. Numerical variables were recorded as means within each group and an independent t-test or Mann Whitney $U$ test was used to test for statistical significance between groups. A p-value of $<0.05$ was deemed significant. IBM SPSS version 26 was used for statistical analysis of the patient population captured within the ASRI

Results: At the time of analysis, physical measurements were available on 692 patients in the ASRI made up of $96.2 \%$ (666) Caucasians with $22.3 \%$ (154) females and $77.7 \%$ (538) males. The average age was 45.9 years with a mean disease duration of 18.8 years (mean scores: BASDAI 4.09, BASFI 3.71, BASMI 3.99, HAQ 0.54, ASQoL 6.72). 80.9\% (560) of patients had radiographic sacroiliitis while $49.1 \%$ (340) had sacroiliitis on MRI.

Overall $29.5 \%$ (215) patients were obese based on BMI results, while $37.5 \%$ (274) were centrally obese as per the WtHpR. Analysis on the basis of sex revealed no significant variation in mean BMI (28.1 vs $27.7, p=0.45)$ or prevalence of obesity as assessed by BMI (29.9\% vs $28.6 \%, p=0.49)$ between males and females(table 1). As expected analysis of $\mathrm{WtHpR}$ revealed higher mean ratios in males compared to females $(0.96$ vs $0.89, \mathrm{p}<0.01)$, however there was a significantly higher prevalence of abdominal obesity in females ( $29.9 \%$ vs $68.2 \%, p<0.01$ ).

Conclusion: There is a high prevalence of abdominal obesity as assessed by $\mathrm{WtHpR}$ in axSpA, this is especially high in females with axSpA. Use of WtHpR should be considered when screening for obesity in this population.

Disclosure of Interests: Sinead Maguire Grant/research support from: Recipient of the Gilead Inflammation fellowship grant, Phil Gallagher: None declared, Fiona Wilson: None declared, Finbar Barry O'Shea: None declared
Table 1. Assessment of Obesity by Sex

\begin{tabular}{lccc}
\hline & Males & Females & p value \\
\hline $\mathbf{n}$ & $77.7 \%(538)$ & $22.3 \%(154)$ & \\
Weight $(\mathrm{kg})$ & 84.98 & 72.76 & $<0.01$ \\
BMI & 28.08 & 27.69 & 0.45 \\
Underweight & $0.9 \%(5)$ & $0.06 \%(1)$ & 0.49 \\
Normal Weight & $29.2 \%(157)$ & $36.4 \%(56)$ & \\
Overweight & $39.2 \%(211)$ & $34.4 \%(53)$ & \\
Obese & $29.9 \%(161)$ & $28.6 \%(44)$ & \\
Waist Circumference $(\mathrm{cm})$ & 97.43 & 90.03 & $<0.01$ \\
Hip Circumference $(\mathrm{cm})$ & 102.11 & 101.27 & 0.56 \\
Waist to Hip Ratio & 0.956 & 0.8901 & $<0.01$ \\
Normal Weight & $23.8 \%(128)$ & $17.5 \%(27)$ & $<0.01$ \\
Overweight & $46.3 \%(249)$ & $14.3 \%(22)$ & \\
Obese & $29.9 \%(161)$ & $68.2 \%(105)$ & \\
\end{tabular}

DOI: 10.1136/annrheumdis-2021-eular.350

\section{POS0950 $\quad$ ARE THERE GENDER DIFFERENCES IN AXIAL SPONDYLOARTHRITIS: DATA FROM A PORTUGUESE SPONDYLOARTHRITIS COHORT}

A. R. Cunha ${ }^{1}$, R. Aguiar $^{1}$, M. J. Santos ${ }^{2}$, V. Afreixo ${ }^{3}$, A. Barcelos ${ }^{1,4,5} .{ }^{1}$ Centro Hospitalar do Baixo Vouga, Rheumatology, Aveiro, Portugal; ${ }^{2}$ Hospital Garcia de Orta, Rheumatology, Almada, Portugal; ${ }^{3}$ University of Aveiro, Department of Mathematics and CIDMA - Center for Research \& Development in Mathematics and Applications, Aveiro, Portugal; ${ }^{4}$ University NOVA, Lisbon, NOVA National School of Public Health, Public Health Research Centre, Lisbon, Portugal; ${ }^{5}$ University NOVA, Lisbon, Comprehensive Health Research Center (CHRC), Lisbon, Portugal

Background: Historically, axial spondyloarthritis (particularly ankylosing spondylitis) was considered a men's disease and has been under-recognized in women.

Emerging evidence reveals gender differences in patophysiology, disease presentation and therapeutic efficacy.

Objectives: To determine if there are differences between genders in a Portuguese cohort of patients with axSpA as regards clinical manifestations, disease activity, functional capacity, patient related outcomes and radiographic findings. Methods: Patients with $\geq 18$ years fulfilling the ASAS-Assessment of Spondyloarthritis International Society classification criteria for axSpA and registered in the electronic national database- Reuma.pt were included in a multicentric cross-sectional study. Sociodemographic data, clinical features and radiographic findings were collected from the first record in Reuma.pt. These variables were compared between genders using Mann-Whitney test and Chi-Square test. Variables with a significant association with group variable (gender) were considered in the multiple variable analysis to adjust the gender effect on the outcome variables.

Results: A total of 1995 patients were included, 1114 (55.9\%) men and 881 $(44.1 \%)$ women. Men had a lower median age at onset of disease (25.1 vs 28.4 $\mathrm{p}=0.000)$ and median age at diagnosis $(26.9$ vs $30.4, \mathrm{p}=0.000)$ and were more frequently smokers $(32.1 \%$ vs $15.7 \%, p=0.000)$. Comparing to women, men had worse BASMI scores (4.0 vs 3.4, $\mathrm{p}=0.000$ ), higher levels of CPR (10.5 vs 6.9 , $\mathrm{p}=0.000)$ and were more often HLA-B27 positive (67.8\% vs $54 \%, p=0.000)$. In univariable analysis, sacroiliitis on radiograph or/and MRI (95.5\% vs $91.7 \%$, $\mathrm{p}=0.04$ ) was more common in men, however that wasn't confirmed in multivariable analysis.

In contrast, women more frequently had more inflammatory bowel disease (8.8\% vs $4.9 \%, p=0.004)$, higher levels of $E S R(25.0$ vs $21.0, p=0.003)$ and worse PROs- BASDAI (5.7 vs 4.5, $\mathrm{p}=0.000), \mathrm{PGA}(60.0$ vs $55.0, \mathrm{p}=0.000)$ and fatigue (6.2 vs $5.4, p=0,000)$

Conclusion: Physicians must be aware of differences between genders in axial spondyloarthritis because this can result in less underdiagnosis and misdiag nosis, allow optimization of treatment strategies, and decrease overall disease burden in women with axSpA patients.

\section{REFERENCES:}

[1] Rusman T, van Vollenhoven RF, van der Horst-Bruinsma IE. Gender Differ ences in Axial Spondyloarthritis: Women Are Not So Lucky. Curr Rheumato Rep. 2018;20(6):35.

[2] Tournadre A, Pereira B, Lhoste A, Dubost JJ, Ristori JM, Claudepierre P, et al. Differences between women and men with recent-onset axial spondyloarthritis: results from a prospective multicenter French cohort. Arthritis Care Res (Hoboken). 2013;65(9):1482-9.

Disclosure of Interests: None declared

DOI: 10.1136/annrheumdis-2021-eular.497 